\title{
Asthmarisiko für Frühgeborene erhöht
}

\author{
Eine epidemiologische Studie sollte klären, ob frühge- \\ borene Säuglinge in der späten Kindheit, im Jugend- \\ und späteren Erwachsenenalter öfter ein Asthma bron- \\ chiale entwickeln als mit regelhaftem Gestationsalter \\ Geborene.
}

- In einer Geburtenkohortenstudie in Schweden wurden über 600000 Neugeborene aus den Jahren 1973-1979 über 25,5 bis 35 Jahre nachbeobachtet. Ein Asthma wurde bei der Verschreibung eines Beta-2-Agonisten und eines inhalativen Kortikosteroids oder eines anderen Atemwegspräparats angenommen. Nur extreme Frühchen (23.-27. Gestationswoche) hatten eine gesteigerte Asthmawahrscheinlichkeit. Das Risiko war in dieser Gruppe 2,4-fach erhöht. Höhere Gestationswochen und eine Fülle von anderen Faktoren, z.B. der soziale Status der Mutter, eine vorherige hormonbasierte Kontrazeption, eine Asthmamedikation der Mutter oder das Familieneinkommen prädisponierten nicht für eine Asthmaentwicklung.

\section{Kommentar}

Seit Längerem ist bekannt, dass Frühgeborene für asthmaähnliche Symptome in der späteren Kindheit prädisponiert sind. Diese Studie imponiert durch ihre Größe und die lange Beobachtungszeit. Limitierungen sind die Asthmadefinition, die nur auf einer kombinierten Atemwegsmedikation (und nicht auf einer Lungenfunktionsprüfung) fußt und die damit mögliche Selektion für höhere Schweregrade, die Wahrscheinlichkeit einer durch die Frühgeburt bedingten intensiveren ärztlichen Betreuung, die auch eine höhere Verschreibungsrate induziert haben könnte, und/oder die Abschätzung des Gestationsalters nach Patientenunterlagen, nicht jedoch z.B. nach einer Ultraschallanalyse.

Ungeachtet dieser methodischen Einschränkungen, die sich bei epidemiologischen Studien dieser Größenordnung nicht vermeiden lassen, belegt diese Analyse, dass eine besonders frühe Frühgeburt u.a. auch ein Risikofaktor für ein späteres Asthma ist.

A. GILLISSEN -

\section{- C. Crump et al.}

Risk of asthma in young adults who were born preterm: a Swedish national cohort study.

Pediatrics 127 (2011) e913-920 